\title{
An emergency Altemeier procedure for necrosis of full-thickness rectal prolapse
}

\author{
Thibault De Schlichting $^{1} \cdot$ Gilles Poncet $^{1,2} \cdot$ Paul Bufacchi $^{1} \cdot$ Arnaud Pasquer $^{1,2}$ (])
}

Received: 10 April 2021 / Accepted: 5 May 2021 / Published online: 11 May 2021

(c) Springer Nature Switzerland AG 2021

Full-thickness external rectal prolapse is a frequent pathology in the elderly population. Patients often complain about anal pain, recurrent prolapse, pelvic trouble, dyschezia and fecal incontinence [1]. Strangulation of full-thickness prolapse is a rare but dreaded complication

We present here the case of a 78-year-old patient who was initially scheduled for a laparoscopic ventral rectopexy indicated because of full-thickness prolapse associated with severe fecal incontinence (Wexner score was 18/20). The intervention was delayed due to COVID-19 restrictions. Finally, on the scheduled day, the patient presented with a strangulated and asymptomatic rectal prolapse with necrosis of its summit. We present here the surgical management of a distal necrosis with the Altemeier procedure [2].

The technical points are presented in the attached video.

The first part of the procedure consisted of identification of the dentate line. Adrenaline was injected in the submucosal plane $1-2 \mathrm{~cm}$ from the dentate line to decrease the risk of bleeding during dissection. The rectum was sectioned circularly on this line until the Douglas reflection was seen. The second step was to open the Douglas reflection to extract the colon and rectum. The third step consisted of sigmoidectomy through a perineal approach. The mesorectum and mesosigmoid were divided with thermofusion device. In the fourth step, the sigmoid colon was pulled through the anal margin and posterior perineorraphy was performed. In the fifth step, sigmoidotomy was performed on the anterior part and absorbable stiches were placed circularly to perform a coloanal anastomosis.

The postoperative course was uneventful.

Arnaud Pasquer

arnaud.pasquer@chu-lyon.fr

1 Department of Digestive and Oncologic Surgery, Edouard

Herriot University Hospital, Lyon, France

2 Claude Bernard Lyon 1 University, Lyon, France
After an initial period of persistent fecal incontinence, the patient had physiotherapy for 3 months. At 6 months, the Wexner score was 5/20.

TCOL-D-21-00,194(Pasquer) video link. https://we.tl/tkp9TA4tW62.

Supplementary Information The online version contains supplementary material available at https://doi.org/10.1007/s10151-021-02462-3.

Authors' contributions Manuscript content warranty: AP, PG, TDS, BP; study design: AP, PG, TDS, BP; manuscript revision: AP, PG, TDS, BP; reference research: AP, PG, TDS, BP.

Declaration

Conflict of interest The authors declare that they have no conflict of interest.

Ethical approval Approval procedures performed in studies involving human participants were in accordance with the ethical standards of the institutional and/or national research committee and with the 1964 Helsinki declaration and its later amendments or comparable ethical standards.

Informed consent Informed consent was obtainer from all individual participants included in the study.

\section{References}

1. Bordeianou L, Hicks CW, Kaiser AM, Alavi K, Sudan R, Wise PE (2014) Rectal prolapse: an overview of clinical features, diagnosis, and patient-specific management strategies. J Gastrointest Surg 18(5):1059-1069

2. Altomare DF, Binda GA, Ganio E, Nardi PD, Giamundo P, Pescatori M (2009) Long-term outcome of Altemeier's procedure for rectal prolapse. Disease of the Colon Rectum 52(4):698-703

Publisher's Note Springer Nature remains neutral with regard to jurisdictional claims in published maps and institutional affiliations. 